\title{
Frieden und Globalisierung
}

\section{Gerald Schneider}

Krieg und Frieden, Friedens- und Konfliktforschung, Frieden in den Theorien der Internationalen Beziehungen, Frieden und Demokratie, Frieden im Inneren, Frieden und Wirtschaft

\section{Einleitung}

2 Der Freihändlerische Liberalismus: Definitionen und mögliche Alternativen

3 Die innere Friedensdividende: Binnenstaatliche Konsequenzen der Globalisierung

4 Die äußere Friedensdividende: Zwischenstaatliche Gewalt und Ökonomische Integration

5 Protektionismus und Politische Gewalt

\section{$1 \quad$ Einleitung}

"What an extraordinary episode in the economic progress of man that age was which came to an end in August 1914!" So elegisch bewertete vor hundert Jahren John Maynard Keynes (1919: 9) die Wohltaten der Globalisierung, welche seiner Meinung nach die Zeit vor dem 1. Weltkrieg geprägt hatten. Für den privilegierten Teil seiner Zeitgenossen sei es in dieser Periode selbstverständlich gewesen, während des Morgentees telefonisch die verschiedensten Köstlichkeiten der Welt zu bestellen. Doch unscheinbare „Schlangen“ wie der Militarismus und der Protektionismus hätten das Paradies der ökonomischen Integration zerstört (ebenda: 10).

Die liberale Position, wonach die Globalisierung Wohlstand schafft und dadurch das Potential für militärische Gewalt reduziert, hatte Norman Angell (1909) bereits zehn Jahre zuvor auf den Punkt gebracht. So meinte der spätere Friedensnobelpreisträger, die zunehmende ökonomische Interdependenz hätte Krieg zwecklos gemacht. ${ }^{1}$ Er nahm damit

1 Angell wurde nach dem 1. Weltkrieg vorgeworfen, mit dieser These eine der größten Fehlprognosen geleistet zu haben. Tatsächlich hat er aber nicht erklärt, dass Krieg undenkbar geworden sei (Schneider 2014). 
eine Position auf, die seit der Aufklärung neben der These des „Demokratischen Friedens“ zu den zentralen Postulaten jenes Theoriestranges gehört, der sich in den Internationalen Beziehungen über die Jahrhunderte hinweg zum sog. Liberalismus entwickelte. Während bereits Montesquieu in seinem zentralen Werk De l'esprit des lois (1748, nach Spector 2010: 64) dem freihändlerischen Geist die Tendenz zur „Mäßigung“ attestiert, kann nach Kant ([1795] 2013: 44) „der Handelsgeist (...) mit dem Kriege nicht zusammen bestehen“. Nach Russett und Oneal (2001) ist ökonomische Integration neben Demokratie und der Zusammenarbeit eine der drei Stützen des Kantischen „Dreifußes des Friedens“.

Natürlich haben Politiker diese Gedanken immer wieder aufgenommen, um den Handel und die daraus folgende Befriedung von Staaten zu propagieren. So meinte etwa der Singapurer Autokrat Lee Kuan Yew 1993, dass die Alternative zu Freihandel nicht nur Armut, sondern auch Krieg sei (Economist 1993: 24). Die These des freihändlerischen Liberalismus, wonach es eine „Friedensdividende“ der Globalisierung gebe, ist jedoch immer wieder auf Kritik gestoßen. Besonders die kanadische Journalistin Naomi Klein (2007:343) hat in verschiedensten Schriften die These vertreten, dass Freihandel im Gegenteil Krieg bedeute - ohne je auf die ideengeschichtlichen Vorläufer dieser These wie etwa Galtungs (1971) strukturelle Theorie des Imperialismus einzugehen. Gerade die neoliberalen Interventionen des Internationalen Währungsfonds verwandelten ihrer Ansicht nach „Krisen in Katastrophen “. Wesentlich differenzierter als solche Generalabrechnungen wirken im Vergleich dazu Globalisierungskritiker wie Stiglitz (2002), der nicht den Freihandel, wohl aber die Deregulierung des Kapitalverkehrs als destabilisierenden Faktor begreift.

Dieses Kapitel stellt die Hauptthese des freihändlerischen Liberalismus vor und qualifiziert ähnlich wie Stiglitz, dass Globalisierung eine Gesellschaft tatsächlich befrieden, dass aber gleichzeitig Liberalisierung ohne Kompensation der Verlierer der Transition das Konfliktrisiko steigern kann (Bussmann/Schneider/Wiesehomeier 2005; Bussmann/ Schneider 2007). Ich unterscheide dabei zwischen der inneren und der äußeren „Friedensdividende“. Während Globalisierung längerfristig die Beziehungen innerhalb eines Staates dank dem erhöhten Wirtschaftswachstum friedlicher macht, erhöht sie für Regierungen die Opportunitätskosten von zwischenstaatlichen Eskalationsprozessen trotz der Effizienzgewinne, die mit der außenwirtschaftlichen Öffnung einhergehen (Polachek 1980) und die sich auch in erhöhte Rüstungsanstrengungen umsetzen lassen. Aufgrund dieser Argumente und der damit verknüpften empirischen Evidenz erwarte ich im Umkehrschluss von der derzeit durchaus möglichen Abkehr von der Globalisierung ein erhöhtes Risiko für organisierte politische Gewalt. 


\section{Der Freihändlerische Liberalismus: Definitionen und mögliche Alternativen}

Der Freihändlerische Liberalismus ist ein zentrales Friedenskonzept der Aufklärung. Es besagt, dass Handel und andere Formen des ökonomischen Austausches Staaten im Inneren wie auch im Verkehr mit anderen Nationen befrieden (Russett/Oneal 2001; Schneider 2014). Da der Begriff des Freihandels nicht alle relevanten Dimensionen der Außenwirtschaftspolitik umfasst, hat sich die Forschung in den letzten Jahren vor allem auf die friedensfördernde Wirkung der Globalisierung konzentriert. In liberaler Warte sollte dieser Prozess der Entgrenzung eine positive Wirkung haben, die ich anderswo zusammen mit meinen Ko-Autoren als „Friedensdividende der Globalisierung“ bezeichnet habe (Bussmann/Scheuthle/Schneider 2003).

Globalisierung ist ein facettenreicher Begriff, der auch eine kulturelle und soziale Komponente umfasst. Die Friedens- und Konfliktforschung hat sich bis jetzt vor allem auf die wirtschaftliche Dimension dieses Prozesses bezogen. In diesem Sinne diskutiere ich im Folgenden, welche Wirkung die außenwirtschaftliche Öffnung bzw. Offenheit hat (Bussmann/Schneider/Wiesehomeier 2005, Bussmann/Schneider 2007). Es ist also bei der Analyse der Auswirkungen der Globalisierung zwischen dem Niveau (Offenheit) und dem Weg dahin (Öffnung) zu unterscheiden. Ferner spielt es eine Rolle, wie offen ein Land für fremde Güter und Dienstleistungen auf der einen Seite und für ausländisches Kapital auf der anderen Seite ist. Dies bedeutet, dass zwischen der Globalisierung des Handels- und des Kapitalverkehrsregimes zu unterscheiden ist.

Ferner sollten wir zwischen dem regulativen Input und dem ökonomischen Output der Globalisierung unterscheiden. So lässt sich etwa mit Daten des Internationalen Währungsfonds erfassen, wie dereguliert der Freihandel und der Kapitalverkehr in einem Land rein juristisch ist (Bodenstein/Plümper/Schneider 2002; Martin/Schneider 2007). Der ökonomische Output bezieht sich auf die Importe bzw. Exporte eines Landes (Handelsregime) bzw. die Direktinvestitionen, die ein Land empfängt oder tätigt (Kapitalverkehr). Investitionen können dabei kurz- oder langfristig sein. Einen ausgedehnten Zeithorizont haben etwa Geldgeber, die Kapital in Produktionsstätten in einem anderen Land einschießen. Solche langfristigen Direktinvestitionen sind vom Kauf von ausländischen Wertpapieren zu unterscheiden. Die sog. Portfolioinvestitionen sind nach der Deregulierung der Finanzmärkte in den 1980er und 1990er Jahren explosionsartig gewachsen. Sie sind wohl die wichtigste Komponente der ökonomischen Integration in der zweiten Hälfte des 20. Jahrhunderts (Bartsch/Schneider 2011).

Länder, die sich freiwillig oder auf Empfehlung von internationalen Institutionen ihre Außenwirtschaft liberalisieren, sind nicht zwangsläufig offen. So können einerseits nichttarifäre Handelshemmnisse den freien Verkehr von Waren und Dienstleistungen trotz aller Globalisierungsbekundungen aus der Politik behindern oder unterbinden, und andererseits Diskriminierungen im Kapitalverkehr internationale Investoren davon abschrecken, in einem Land wirtschaftliche Verantwortung zu übernehmen. Ohnehin ist $\mathrm{zu}$ bedenken, dass kleine Volkswirtschaften in der Regel offener sind als große Länder. 
Die ausgeprägte Globalisierung der Kleinen ist der Not geschuldet, nicht für einen großen eigenen Binnenmarkt produzieren zu können.

So eingängig der Begriff der Globalisierung an sich ist, so schwierig ist zugleich die Messung seiner Hauptkomponenten. So spielt es etwa eine Rolle, ob die aufaddierten Exporte und Importe durch das Bruttoinlandprodukt oder durch die Bevölkerungsgröße geteilt werden. Andere Probleme bestehen in der beschränkten Verfügbarkeit von Daten beispielsweise zu Direktinvestitionen. Dazu kommt, dass politische Handlungen oft einem viel kürzeren Zeithorizont unterworfen sind als wirtschaftliche Entscheidungsprozesse. So können Unternehmer bei Ausbruch einer Krise nicht so schnell die langfristigen Investitionen aus einem Land abziehen, in dem die Politik den Befehl zu einem Waffengang erteilt hat. Dies kann bedeuten, dass wir die Kosten einer aggressiven Außenpolitik oft deshalb unterschätzen, weil die früheren Investitionsentscheidungen sich nicht so leicht rückgängig machen lassen. Die begrenzte Aussagekraft von Handelsdaten haben Schneider und Tröger (2006) dazu bewogen, Kursschwankungen an internationalen Finanzmärkten in die Konfliktforschung einzubeziehen, um so präziser den Einfluss von Gewalt auf die Wirtschaft abzuschätzen.

In theoretischer Hinsicht spielt auch eine Rolle, ob wir die friedensfördernden Effekte eher von der außenwirtschaftlichen Orientierung eines Staates oder von dessen kapitalistischer Prägung erwarten. Gartzke (2007), Mousseau (2000, 2013), McDonald (2009) und Weede (1995) propagieren unterschiedliche Varianten des „Kapitalistischen Friedens“ und behaupten vereinzelt, dass dieser Erklärungsstrang andere liberale Theorien wie etwa die These des Demokratischen Friedens ersetze. Verschiedene Replikationsstudien zeigen aber deutlich, dass sich dieser Alleinvertretungsanspruch für diese alternative liberale Theorie nicht aufrechterhalten lässt (Dafoe 2011, Dafoe/Oneal/Russett 2013). Schwieriger wiegen nach meiner Sicht aber die konzeptuellen Probleme, die die Theorie des kapitalistischen Friedens seit ihrer Skizze durch Schumpeter (1918/9) prägen. So mangelt es der Theorie an einer klaren und schlanken Definition der Attribute, die den Kapitalismus auszeichnen (Schneider/Gleditsch 2010, Schneider 2017). So reicht die Spanne der Vorstellungen zum Kern dieser Wirtschaftsordnung von einem Hayekianischen Minimalstaat (McDonald 2009) bis hin zum Wohlfahrtsstaat, in dem die Bürger dank der Dichte von Verträgen einander vertrauten (Mousseau 2013). Ferner betont keine der Theorien des Kapitalistischen Friedens jenes anarchische Element, welches sowohl Marx wie Schumpeter als Wesensmerkmal einer kapitalistischen Ordnung ansahen. So gehört für die zwei so unterschiedlichen Theoretiker das Auf und Ab zwischen Booms und Rezessionen einfach zum Risiko dieser Art der volkswirtschaftlichen Organisation (Schneider 2017). Solange die Theorie des Kapitalistischen Friedens die Einbrüche der Wirtschaftsentwicklung, die den Kapitalismus prägen, nicht berücksichtigt und als reine Schönwetterthese daher kommt, stellt sie keine Alternative zum freihändlerischen Liberalismus dar. 


\section{Die innere Friedensdividende: Binnenstaatliche Konsequenzen der Globalisierung}

Die Differenzierung zwischen Offenheit und Öffnung als sich ergänzende Aspekte der Globalisierung hat der Autor in Zusammenarbeit mit Margit Bussmann genutzt, um den freihändlerischen Liberalismus zumindest für die innere Stabilität eines Landes zu qualifizieren (Bussmann/Schneider 2007). Allerdings besteht die innere Friedensdividende nur für die außenwirtschaftliche Offenheit, nicht aber für deren Liberalisierung.

Unbestritten ist in der Warte der Neoklassik, dass Freihandel wie auch Kapitalverkehrsfreiheit die Wohlfahrt eines Staates erhöhen. Für diesen positiven Effekt der außenwirtschaftlichen Offenheit sind beim Handel nach Ricardo die Effizienzgewinne verantwortlich, die mit der Spezialisierung auf die Produktion von Gütern einhergeht, in der eine Volkswirtschaft einen komparativen Vorteil besitzt. Die Öffnung eines Staates für ausländische Direktinvestitionen erhöht den Kapitalstock eines Landes und damit ebenso dessen Wachstumspotential.

Schwieriger wird die Analyse, wenn wir die Friktionen in Betracht ziehen, die bei einer außenwirtschaftlichen Öffnung zu erwarten sind. So bestehen in der Perspektive des Ricardo-Viner-Modells Mobilitätshindernisse in der kurzen Frist für Produktionsfaktoren, die an ein importkonkurrierendes Unternehmen gebunden sind, das aufgrund der Globalisierung schrumpft oder vom Markt verschwindet. Der Prozess der „kreativen Zerstörung“, der nach Schumpeter (1942) das Wesensmerkmal des Kapitalismus ist, vernichtet Kapital und Arbeitsplätze. Die daraus entstehende Frustration kann, wenn die Krise tief genug reicht und die Wege zum friedlichen Protest verbaut sind, in dieser Perspektive auch in Gewalt umschlagen.

In der langen Frist eignet sich das Ricardo-Viner-Modell allerdings wenig, um die sozialen Spannungen um die außenwirtschaftliche Öffnung zu verstehen. So flaut die Konfliktlinie zwischen der importkonkurrierenden Industrie und dem Exportsektor mit der Zeit ab, da freigestellte Arbeitskräfte aus nicht wettbewerbsfähigen Industrien sich wieder in florierenden Unternehmen bewerben können, die dank der Öffnung zusätzliche Arbeitsplätze geschaffen haben. Relevant für das langfristige Verständnis der Konflikte ist vor allem das Heckscher-Ohlin-Modell, das die Auseinandersetzung um die Globalisierung als Konflikt zwischen Produktionsfaktoren sieht. So ist jeweils jener Faktor freihändlerisch eingestellt, der im Überschuss vorhanden ist. Dies ist der Faktor Kapital in den Industrie- und der Faktor Arbeit in den Entwicklungsländern. Diese klassenkämpferische Makroperspektive wird im Heckscher-Ohlin-Modell um die individualistische Perspektive ergänzt, dass eine bessere Ausbildung mit einer positiveren Einstellung gegenüber der Globalisierung einhergehen sollte: Arbeitskräfte mit höherem Humankapital sind insofern als Gewinner offener Grenzen zu betrachten, als sie sich so eher dem Lohndiktat in geschützten Sektoren entziehen können. Weitere Qualifizierungen ergeben sich über die Veränderungen in der Arbeitswelt. So hängt in den Industriestaaten das Konfliktpotential um die außenwirtschaftliche Öffnung immer stärker davon an, ob der Job einer Arbeitskraft ins Ausland ausgelagert ist oder durch Roboter ersetzt zu werden droht (Owen/Johnston 2017). Ferner 
spielt es eine Rolle, welche Art von Kapital in ein kapitalhungriges Land fließt. Wenn ein Land wirtschaftlich unter Druck gerät, können Geldgeber besonders Wertpapieranlagen kurzfristig abziehen und so nach Stiglitz (2002) die Stabilität von Staaten gefährden.

Bussmann und Schneider (2007) zeigen in einer globalen Analyse in Übereinstimmung mit ihrer Qualifikation der liberalen Friedenshoffnungen, dass die Liberalisierung des Außenhandels die Konfliktneigung fördert. Damit ist die innere Friedensdividende den bereits globalisierten Staaten vorbehalten, aber nicht jenen, die im Prozess der Öffnung begriffen sind. Magee und Massoud (2011) können diese These mit einem leicht veränderten Forschungsdesign bestätigen. Sie identifizieren den Umschlagepunkt einige Jahre vor der Globalisierungswelle, da die möglichen Liberalisierungsmaßnahmen ja zunächst geplant werden müssen und Proteste gegen die Öffnung besonders dann Sinn machen, wenn diese noch nicht formell beschlossen ist. Zu berücksichtigen ist auch, dass diese Effekte je nach Sektor stärker oder schwächer ausfallen können. Zur Instabilität kann im Rohstoffsektor etwa beitragen, dass internationale Investoren aufgrund des beschränkten Zeithorizontes für ihr Engagement oder aus Angst vor Enteignung weniger am Gedeihen der Region interessiert sind, in der eine Mine oder ein Erdölfeld liegt. Dies erklärt nach Wegenast und Schneider (2017), warum im Rohstoffsektor der Ressourcenfluch lokal eher von internationalen als von nationalen Investoren ausgeht.

Die Friedensdividende der Globalisierung lässt sich aber realisieren, wenn Regierungen den wirtschaftlichen Wandel durch sozialpolitische Maßnahmen abfedern. So hat Burgoon (2006) gezeigt, dass sich Staaten gegen transnationalen Terrorismus durch wohlfahrtsstaatliche Maßnahmen absichern können. In Entwicklungsländern geht eine Reduktion des binnenstaatlichen Terrorismus mit der Vergabe gezielter Entwicklungshilfe etwa für die Zivilgesellschaft einher (Savun/Tirone 2018), und Genovese, Schneider und Wassmann (2016) zeigen, dass in Ländern, deren Stabilität durch Austeritätsmaßnahmen bedroht ist und bei denen die fiskalische Krisenlinderung versagt, unorthodoxe geldpolitische Instrumente ebenfalls eine befriedende Wirkung entfalten können.

\section{Die äußere Friedensdividende: Zwischenstaatliche Gewalt und Ökonomische Integration}

Für zwischenstaatliche Beziehungen besteht die Grundlage für die Friedensdividende der Globalisierung in den wachsenden Opportunitätskosten, welche die außenwirtschaftliche Verflechtung schafft. Die Logik dieses Argumentes besagt, dass eine Regierung ihrer Wirtschaft höhere Kosten durch eine aggressive Außenpolitik aufbürdet, je stärker dieses Land sich wirtschaftlich geöffnet hat. Dies schreckt die Entscheidungsträger nach Polachek (1980), Rosecrance (1986) oder Russett und Oneal (2001) davon ab, auf eine handelszerstörende Außenpolitik zu setzen. Eine solche Annahme hat aber den Nachteil, dass dadurch die Kriegsführung zum irrationalen Akt verkommt, da sich ein Waffengang nicht nur ex post, sondern auch ex ante nicht lohnt (Schneider 2014). Ferner ist ein Konflikt zwischen 
Staaten, die nicht integriert sind, eher unwahrscheinlich, da diese möglichen Kontrahenten ja gar keinen gewichtigen Streitgrund haben, der einen Waffengang motivieren könnte. Aus diesem Grund ist anzunehmen, dass der Zusammenhang zwischen Interdependenz und Konflikt eher krummlinig statt linear verläuft, also dass sowohl nicht integrierte als auch besonders stark integrierte Volkswirtschaften eine geringe Konfliktwahrscheinlichkeit aufweisen, während bei schwacher oder partieller ökonomischer Interdependenz das Konfliktrisiko am höchsten ist.

Auf empirischem Feld war die Literatur in den 1990er Jahren besonders durch den Disput zwischen Barbieri (1996, 2002) und Oneal und Russett (Oneal/Maoz/Russett 1996, Russett/Oneal 2001) geprägt. Barbieri konnte zunächst für die Analyse von Staatenpaaren zeigen, dass dort, wo die Handelsinterdependenz hoch ist, das Konfliktrisiko am höchsten ist. Doch das statistische Modell der Autorin genügte nicht höchsten Ansprüchen. Nach Jun, Xu und George (2007) dämpft Interdependenz das Konfliktrisiko, wenn man für Machtfaktoren in der Analyse kontrolliert. Insgesamt überwiegen in den empirischen Analysen diese positiven Befunde deutlich, auch wenn die Art der Handelsbeziehungen in die Analyse einzubeziehen ist und unterschiedliche Eskalationsstufen zu berücksichtigen wären. Nach Coglan (2013) spielt es aber auch eine Rolle, welche Art von Gütern exportiert wird. Ölexporteure mit radikaler Ideologie initiieren öfters Kriege als andere Staatslenker.

Eine weitere Qualifizierung der äußeren Friedensdividende der Globalisierung erwächst aus den Koalitionen, welche die außenwirtschaftliche Offenheit und eine aggressive Außenpolitik gleichzeitig stützen. Schneider und Schulze (2003) können für ein Land mit drei Gruppen (dem Militär, der Export- und der importkonkurrierenden Industrie) formaltheoretisch zeigen, dass der Einfluss der Globalisierung zunächst auch Konflikte auf einem tieferen Eskalationsniveau begünstigt. Dies rührt daher, dass die ökonomische Integration die Einkünfte des Exportsektors in die Höhe treibt und über die Vergrößerung der Steuerbasis auch die Steuereinnahmen zunehmen, welcher der Staat für eine aggressive Außenpolitik braucht. Nach dieser Logik unterstützt der Exportsektor die vom Militär geforderte Aggressionen bis zu dem Punkt, an dem die Kosten dieser Politik größer sind als der Gewinn, der mit der Globalisierung verknüpft ist.

Eine weitere Einschränkung der freihändlerischen These besteht nach Martin, Mayer und Thoenig (2008) darin, dass sich die Abschreckungswirkung der Interdependenz nur in bilateralen Handelsbeziehungen, nicht aber in einem multilateralen Netzwerk entfalten kann. Der Grund für diese Diskrepanz liegt darin, dass Handelspartner in einem multilateralen Netzwerk leicht substituiert werden können, wenn sie wegen eines Konfliktes wegfallen. Die Gewinne, die insgesamt aus Freihandel entstehen, lassen sich dann in dieser Perspektive auch für die eigene Aufrüstung verwenden.

Damit schließt sich der Kreis hin zu den realistischen Theoretikern, die im Nachgang zur Grundlegung des strukturellen Realismus eine befriedende Wirkung von Handel ausschlossen, weil Staaten durch den Handel mit einem Konkurrenten dessen zusätzliche Aufrüstungsinvestitionen fürchten müssen (Grieco 1990). Empirisch ist diese grobschlächtige These der relativen Gewinne längst wiederlegt, halten doch Staaten selbst im Konfliktfall 
oft ihre Handelbeziehungen aufrecht bzw. lässt sich langfristig angelegtes Kapital nicht so leicht aus einem anderen Staat abziehen.

Warum sich Direktinvestitionen befriedend auf die Staatenbeziehungen auswirken sollten, ist theoretisch weniger klar entwickelt, aber empirisch klar erwiesen (Bussmann 2010). Aber auch hier entstehen Opportunitätskosten, und zwar beim Investor wie beim Investitionsempfänger, wenn eine Regierung Spannungen zu einem anderen Staat schafft. Ähnliche lindernde Wirkungen gehen von ökonomischer Interdependenz auf das Ausmaß des transnationalen Terrorismus aus, dem ein Staat ausgesetzt ist (Li und Schaub 2004). Zudem dämpft die ökonomische Integration von Staaten das Risiko von Genoziden (Harff 2003) und der Abschluss von Freihandelsverträgen verbessert auch die Menschenrechtssituation in den Staaten, die sich so gegenseitig binden (Hafner-Burton 2005, 2009, für eine Kritik siehe Spilker/Böhmelt 2013).

Trotz der insgesamt zumeist klaren empirischen Evidenz zugunsten des freihändlerischen Liberalismus ist zu bemängeln, dass er die Mikromechanismen bis jetzt nicht klar herausgearbeitet hat. Während die Literatur zum Demokratischen Frieden die Rolle der Regierung und Opposition in der Krisenentscheidungsfindung modelliert hat (Schultz 2001), fehlen in den meisten Beiträgen zur Globalisierungsdiskussion die ökonomischen Entscheidungsträger. Es ist möglich, wie Gartze (2007) formuliert, dass die Finanzbranche in Zeiten des Konfliktes den Handlungsträgern in der Politik klare Signale sendet, doch dieser kausale Mechanismus ist in der spieltheoretischen Analyse identisch mit jenem, der zugunsten des Demokratischen Friedens vorgebracht wird (Schneider 2014).

\section{$5 \quad$ Protektionismus und Politische Gewalt}

Bei einer Theorie wie der These des freihändlerischen Friedens ist es nicht automatisch so, dass die Abwesenheit einer Bedingung wie der Globalisierung einen gegenläufigen Effekt hat. Dennoch ist zu vermuten, dass Protektionismus und die mit ihm verknüpften wirtschaftlichen Schrumpfungsprozesse Konflikte wahrscheinlicher machen, selbst als ein besonders ausgeprägter Globalisierungsschub. Wirtschaftshistoriker wie Kindleberger (1973) haben einen breiten Bogen gespannt von den Handelskriegen der Zwischenkriegszeit, dem Ausbruch der Großen Depression und dem Erstarken des Faschismus, die in dieser Perspektive alle ursächlich am Ausbruch des 2. Weltkriegs beteiligt waren.

Überraschend ist in dieser Perspektive aber, dass es bis jetzt keinen klaren vergleichenden Nachweis zum Zusammenhang zwischen Protektionismus und Gewalt gibt. Dass Protektionismus aber sowohl im Innern wie im Äußern ein gewaltiges Konfliktpotential schafft, lässt sich aus der Literatur zu Preisschocks ableiten. Miguel et al. (2004, siehe Ciccone 2011 für eine Kritik) zeigen in einem einflussreichen Aufsatz, dass schwere ökonomische Krisen das Bürgerkriegsrisiko erhöhen können. Für Entwicklungsländer sind besonders Preisschocks in den Grundnahrungsmitteln verheerend. Arezki und Brückner (2011) konnten nachweisen, dass ein gravierender Preisanstieg zu mehr politischer Gewalt 
führt. Wenn die Rohstoffexportpreise anziehen, befrieden sich Gesellschaften hingegen (Brückner/Ciccone 2010).

Ross (2012) argumentiert ähnlich, dass der Preiszerfall von Öl in wichtigen Ölexportierenden Ländern Unruhe schafft, weil die Regierungen zunehmend in Schwierigkeiten geraten, öffentliche Güter zu finanzieren und die Günstlinge des Regimes an der Rohstoffbonanza teilhaben. Gerade der Einbruch der Ölpreise beruhte auf einem Wirtschaftseinbruch in den Industrieländern, welche weniger produzierten und deshalb auch weniger Rohstoffe als Input brauchten.

Aus dieser Perspektive ist der grassierende Neoprotektionismus in den Industrieländern auch aus friedenspolitischer Warte brandgefährlich. Weil die Abschottung Umverteilungskonflikte häuft und die Opportunitätskosten für politische Gewalt senkt, drohen uns auch aus sicherheitspolitischer Perspektive düstere Zeiten. Der Politik ist dabei aber auch ins Stammheft zu schreiben, dass es die Friedensdividende der Globalisierung nicht gratis gibt und dass es zu ihrer Absicherung eine Kompensation an die kurz- und mittelfristigen Verlierer braucht.

\section{Literatur}

Angell, Norman (1909): The Great Illusion: A Study of the Relation of Military Power in Nations to their Economic and Social Advantages. London: Heinemann. (Original 1908 als Europe's optical illusion publiziert).

Arezki, Rabah/Brückner, Markus (2011): Food Prices, Conflict, and Democratic Change. International Monetary Fund Working Paper 11/26, Washington, DC.

Barbieri, Katherine (1996): Economic Interdependence: A Path to Peace or a Source of International Conflict? In: Journal of Peace Research, Vol. 33, Nr. 1, S.29-49.

Barbieri, Katherine (2002): The Liberal Illusion: Does Trade Promote Peace? Ann Arbor, MI: University of Michigan Press.

Bartsch, Wiebke/Schneider, Gerald (2011): Globalisierung: Hinweise zur Klärung eines Begriffs. In: Knobloch, Jörg (Hrsg.) (2011): Kinder- und Jugendliteratur in einer globalisierten Welt: Chancen und Risiken. München: Kopaed, S. 20-35.

Bodenstein, Thilo/Plümper, Thomas/Schneider, Gerald (2002): Two Sides of Economic Openness: Non-Tariff Barriers to Trade and Capital Controls in Transition Countries, 1993-2000. In: Communist and Post-Communist Studies, Vol. 36, Nr. 2, S.231-243.

Brückner, Markus/Ciccone, Antonio (2010): International Commodity Prices, Growth and the Outbreak of Civil War in Sub-Saharan Africa. In: Economic Journal, Vol. 120, Nr. 544, S. 519-534.

Burgoon, Brian (2006): On Welfare and Terror. In: Journal of Conflict Resolution, Vol. 50, Nr. 2, S. 176-203.

Bussman, Margit/Scheuthle, Harald/Schneider, Gerald (2003): Die „Friedensdividende“ der Globalisierung: Außenwirtschaftliche Öffnung und innenpolitische Stabilität in den Entwicklungsländern. In: Politische Vierteljahresschrift, Vol. 44, Nr. 3, S. 302-324. 
Bussmann, Margit/Schneider, Gerald/Wiesehomeier, Nina (2005): Foreign Economic Liberalization and Peace: The Case of Sub-Saharan Africa. In: European Journal of International Relations, Vol. 11, Nr. 4, S. 551-579.

Bussmann, Margit/Schneider, Gerald (2007): When Globalization Discontent Turns Violent: Foreign Economic Liberalization and Internal War. In: International Studies Quarterly, Vol. 51, Nr. 1, S.79-97.

Bussmann, Margit (2010): Foreign Direct Investment and Militarized International Conflict. In: Journal of Peace Research, Vol. 47, Nr. 2, S. 143-153.

Ciccone, Antonio (2011): Economic Shocks and Civil Conflict: A Comment. In: American Economic Journal: Applied Economics, Vol. 3, Nr. 4, S.215-227.

Coglan, Jeffrey D. (2013): Petro-Aggression: When Oil Causes War. Cambridge: Cambridge University Press.

Dafoe, Allan (2011): Statistical Critiques of the Democratic Peace: Caveat emptor. In: American Journal of Political Science, Vol. 55, Nr. 2, S.247-262.

Dafoe, Allan/Oneal, John R./Russett, Bruce (2013): The Democratic Peace: Weighing the Evidence and Cautious Inference. In: International Studies Quarterly, Vol. 57, Nr. 1, S.201-214.

Economist, The (1993): Survey: Asia: A Billion Consumers. In: The Economist, October 30, 5-24.

Galtung, Johan (1971): A Structural Theory of Imperialism. In: Journal of Peace Research, Vol. 8, Nr. 2, S. 81-118.

Gartzke Erik (2007) The Capitalist Peace. In: American Journal of Political Science, Vol. 51, Nr. 1, S. 166-191.

Gartzke Erik/Lupu, Yonatan (2012): Trading on Preconceptions: Why World War I was not a Failure of Economic Interdependence. In: International Security, Vol. 36, Nr. 4, S. 115-150.

Genovese, Federica/Schneider, Gerald/Wassmann, Pia (2016): The Eurotower Strikes Back: Crises, Adjustments and Europe's Austerity Protests. In: Comparative Political Studies, Vol. 49, Nr. 7, S. 939-967.

Grieco, Joseph (1990) Cooperation among Nations: Europe, America, and Non-tariff Barriers to Trade. Ithaca: Cornell University Press.

Hafner-Burton, Emily A. (2005): Trading human rights: How preferential trade agreements influence government repression. In: International Organization, Vo. 59, Nr. 3, 593-629.

Hafner-Burton, Emily A. (2009): Forced to Be Good: Why Trade Agreements Boost Human Rights. Cornell University Press.

Harff, Barbara (2003): No lessons learned from the Holocaust? Assessing risks of genocide and political mass murder since 1955. In: American Political Science Review, Vol. 97, Nr. 1, S.57-73.

Kant, Immanuel ([1795] 2013): Zum ewigen Frieden. Ein philosophischer Entwurf. Stuttgart: Reclam.

Keynes, J.M. (1919): The Economic Consequences of the Peace. London: Macmillan.

Kindleberger, Charles P. (1973): Die Weltwirtschaftskrise. 1929-1939. München: dtv.

Klein, Naomi (2007): The Shock Doctrine: The Rise of Disaster Capitalism. New York: Metropolitan Books.

Li, Quan,/Schaub, Dominic (2004): Economic globalization and transnational terrorism. In: Journal of Conflict Resolution, Vol. 48, Nr. 2, S. 230-258.

Magee, Christopher SP/Tansa George Massoud (2011): Openness and internal conflict. In: Journal of Peace Research, Vol. 48, Nr. 1, S. 59-72.

Martin, Christian W./Schneider, Gerald (2007): Pfadabhängigkeit, Konvergenz oder regulativer Wettbewerb: Determinanten der Außenwirtschaftsliberalisierung, 1978-2002. In: Politische Vierteljahresschrift Sonderheft, Vol. 38, S.449-469.

Martin, Philippe/Mayer, Thierry/Thoenig, Mathias (2008): Make trade not war? In: Review of Economic Studies, Vol. 75, Nr. 3, S. 865-900. 
McDonald, Patrick J. (2009): The invisible hand of peace: Capitalism, the war machine, and international relations theory. New York: Cambridge University Press.

Miguel, Edward/Satyanath, Shanker/Sergenti, Ernest (2004): Economic shocks and civil conflict: An instrumental variables approach. In: Journal of Political Economy, Vol. 112, Nr. 4, S.725-753.

Mousseau, Michael (2000): Market prosperity, democratic consolidation, and democratic peace. In: Journal of Conflict Resolution, Vol. 44, Nr. 4, S. 472-507.

Mousseau, Michael (2013): The democratic peace unraveled: It's the economy. In: International Studies Quarterly, Vol. 57, Nr. 1, S.186-197.

Oneal, John R. et al. (1996): The Liberal Peace: Interdependence, Democracy, and International Conflict, 1950-1985. In: Journal of Peace Research, Vol. 33, Nr. 1, S.11-28.

Owen, Erica/Johnston, Noel (2017). Occupation and the Political Economy of Trade: Job routineness, offshorability and protectionist sentiment. In: International Organization, Vol. 71, Nr. 4, S.665-699.

Polachek, Solomon W. (1980): Conflict and trade. In: Journal of Conflict Resolution, Vol. 24, Nr. 1, S. 55-78.

Rosecrance, Richard (1986): The Rise of the Trading State: Commerce and Conquest in the Modern World. New York: Basic Books.

Ross, Michael (2012): The Oil Curse: How Petroleum Wealth Shapes the Development of Nations. Princeton, NJ: Princeton University Press.

Russett Bruce/Oneal, John R. (2001): Triangulating Peace: Democracy, Interdependence, and International Organizations. New York: WW Norton.

Savun, Burcu/Tirone, Daniel C. (2018): Foreign Aid as a Counterterrorism Tool More Liberty, Less Terror? In: Journal of Conflict Resolution, Vol. 62, Nr. 8, S. 1607-1635.

Schneider, Gerald/Schultze, Günther G. (2003): The domestic roots of commercial liberalism: A sector-specific approach. In: Schneider, Gerald/Barbieri, Katherine/Gleditsch, Nils Petter Gleditsch (eds.): Globalization and Armed Conflict. Lanham, MD: Rowman and Littlefield, S. 103-122.

Schneider, Gerald/Troeger, Vera E. (2006): War and the world economy: Stock market reactions to international conflicts. In: Journal of Conflict Resolution, Vol. 50, Nr. 5, S. 623-645.

Schneider, Gerald/Gleditsch, Nils Petter (2010): The capitalist peace: The origins and prospects of a liberal idea. In: International Interaction, Vol. 36, Nr. 2, S.107-114.

Schneider, Gerald (2014): Peace through Globalization and Capitalism? Prospects of Two Liberal Propositions. In: Journal of Peace Research, Vol. 51, Nr. 2, S. 173-83.

Schneider, Gerald (2017): Capitalist Peace Theory: A Critical Appraisal. In: Thompson, William R. (Hrsg.). The Oxford Research Encyclopedia of Politics. Oxford: Oxford University Press. DOI: 10.1093/acrefore/9780190228637.013.314.

Schultz, Kenneth A. (2001): Democracy and Coercive Diplomacy. New York: Cambridge University Press.

Schumpeter, Joseph Alois (1918-1919): Zur Soziologie der Imperialismen. In: Archiv für Sozialwissenschaft und Sozialpolitik, Vol. 46, S. 1-39 und 275-310.

Schumpeter, Joseph Alois (1942): Capitalism, Socialism and Democracy. New York: Harper.

Spector, Céline (2010): Montesquieu était-il libéral? In: G. Kevorkian (Hrsg): La Pensée libérale. Paris: Ellipses, S. 57-71.

Spilker, Gabriele/Bohmelt, Tobias (2013): The Impact of Preferential Trade Agreements on Governmental Repression Revisited. In: Review of International Organizations, Vol. 8, Nr. 3, S. 343-361.

Stiglitz, Joseph E. (2002): Globalization and its Discontents. London: Allen Lane.

Weede, Erich (1995): Economic policy and international security: Rent-seeking, free trade and democratic peace. In: European Journal of International Relations, Vol. 1, Nr. 4, S.519-537.

Wegenast, Tim/Schneider, Gerald (2017): Ownership Matters: Natural Resources Property Rights and Social Conflict in Sub-Saharan Africa. In: Political Geography, Vol. 61, S. 110-122.

Xiang, Jun/Xiaohan, Xu/Keteku, George (2007): Power: The missing link in the trade-conflict relationship. In: Journal of Conflict Resolution, Vol. 51, Nr. 4, S.646-663. 